\title{
Sistemas de Recomendação em Ambientes Educacionais: Estado da Arte e Perspectivas Futuras
}

\author{
Ramon R. Leite ${ }^{1}$, Cristiano G. Pitangui ${ }^{2}$, Luciana P. de Assis ${ }^{1}$, Alessandro V. Andrade ${ }^{1}$ \\ ${ }^{1}$ Universidade Federal dos Vales do Jequitinhonha e Mucuri (UFVJM) \\ ${ }^{2}$ Universidade Federal de São João Del Rei (UFSJ) \\ \{ramon.leite, lpassis\}@ufvjm.edu.br \\ \{pitangui.cristiano, alessandro.vivas\}@gmail.com
}

\begin{abstract}
The use of Recommendation Systems in the Educational area has been growing in recent years. Such interest is justified since the large amount of content produced from e-learning needs to be filtered and selected to meet users' needs and preferences. The present work reviews the current literature to analyse how the Recommendation Systems are being used in the Educational context. We conducted a research in works from 2016 to 2019, where it was noticed that there are few systems that rely on specific concepts of psychology or pedagogy. It has also been noted that many datasets used to test the systems are not in the public domain. Finally, it was verified that there are techniques that can be better explored, such as the multiobjective recommendation, for example.
\end{abstract}

Resumo. O uso dos Sistemas de Recomendação no ambiente Educacional vem crescendo nos últimos anos. Tal interesse se justifica já que a grande quantidade de conteúdo produzido a partir do e-learning precisa ser filtrada para atender as preferências dos usuários. Este trabalho realiza um estudo dos trabalhos mais recentes da área, no período de 2016 a 2019, com objetivo de apontar o estado da arte atual da pesquisa no tema. Percebeu-se que há poucos sistemas que se apoiam em conceitos específicos da psicologia ou pedagogia. Notouse, ademais, que muitas bases de dados utilizadas para testar os sistemas não são públicas. Por fim, verificou-se que existem técnicas que podem ser melhor exploradas, como a recomendação multiobjetivo, por exemplo.

\section{Introdução}

A grande quantidade de conteúdo disponível na Web faz com que sejam criadas estratégias para filtrar a informação de acordo com as necessidades dos usuários. Um desses mecanismos são os Sistemas de Recomendação, responsáveis por fornecerem aos usuários os itens que de fato correspondam às suas preferências e objetivos [Hassan and Hamada 2016].

[Bobadilla et al. 2013] definem Sistemas de Recomendação como programas que buscam recomendar itens - produtos ou serviços - a usuários, a partir da previsão do interesse dos mesmos. Esse interesse é definido a partir de, por exemplo, informações dos próprios usuários, dos itens disponíveis e das interações entre os usuários e esses itens. 
VIII Congresso Brasileiro de Informática na Educação (CBIE 2019)

Anais dos Workshops do VIII Congresso Brasileiro de Informática na Educação (WCBIE 2019)

[Ricci et al. 2015] afirmam que os Sistemas de Recomendação são ferramentas e técnicas empregadas para sugerir itens aos usuários que os utilizam. Portanto, eles se tornam um meio valioso para se decidir desde o que comprar até o que se ler. Por esse motivo, são amplamente empregados em áreas como comércio eletrônico e serviços de streaming. Um outro campo cuja utilização dos Sistemas de Recomendação vem ganhando destaque é no e-learning. Tendo em vista o crescimento da quantidade de conteúdo disponível nos inúmeros cursos que são criados, ferramentas que selecionem os tipos de itens de acordo com os objetivos e preferências do estudante tornaram-se indispensáveis.

Grande parte do conteúdo disponível no ambiente educacional está organizado sob a forma de Objetos de Aprendizagem (OA). O IEEE (Institute of Electrical and Electronics Engineers) define Objeto de Aprendizagem como "qualquer entidade, digital ou não, que pode ser utilizada para aprendizagem, educação ou capacitação" [IEEE 2002]. Os OAs são compostos basicamente pelo seu conteúdo propriamente dito (texto, áudio, imagem, vídeo, etc.) e pelos metadados que os descrevem (nome, autor, nível de dificuldade, ano de criação, tipo de conteúdo, etc.). Geralmente, esses itens estão organizados em repositórios, os chamados LORs (Learning Objetc Respositories).

De modo a facilitar a reutilização e interoperabilidade de tais repositórios, os OAs costumam seguir determinados padrões dos seus metadados, de forma a descrever o que eles contêm. Alguns desses padrões são IEEE LOM (IEEE standard for learning object metadata) [IEEE 2002], IMS-CP [IMS 2019] e Dublin-CORE [D C M and Others 2008].

Várias pesquisas na área de Sistemas de Recomendação no âmbito Educacional têm sido desenvolvidas. Entre elas, encontram-se os Sistemas de Recomendação de Objetos de Aprendizagem. Muitas abordagens são encaminhadas com o objetivo de recomendar os OAs que melhor correspondam aos anseios do estudante [Santos et al. 2016]. Entre as técnicas, a filtragem colaborativa e a recomendação baseada em conteúdo são muito utilizadas. A primeira consiste em prover a recomendação de itens a um usuário a partir de avaliações que usuários com perfil semelhante fizeram acerca daquele item. Por outro lado, a segunda técnica se baseia nas propriedades semelhantes que um item já utilizado pelo usuário tenha com um outro OA que possa ser recomendado [Sergis and Sampson 2016].

Ambas as abordagens possuem limitações. Quando se possui pouca ou nenhuma informação sobre o usuário, torna-se muito difícil a realização de recomendações. Tal problema é conhecido por cold start. Desse modo, há inúmeras outras estratégias utilizadas para tentar lidar com essa e outras limitações que surgem em cada situação.

Dentre as abordagens encontradas na literatura, podem-se listar os Sistemas de Recomendação baseados em Ontologia, que utilizam um domínio específico de conhecimento no qual o usuário se encontra, para facilitar a identificação das preferências. Há a recomendação por contexto, considerando, por exemplo, o horário e o dia em que se está utilizando o sistema. Há ainda abordagens que aplicam Lógica Fuzzy [Sergis and Sampson 2016], Redes Neurais [Yao 1999], Algoritmo Genético [Katarya and Verma 2018] e Computação Bioinspirada [Hassan and Hamada 2016].

Expostos o contexto e algumas abordagens relacionadas ao tema, o presente trabalho busca compreender, por meio de uma revisão atual da literatura, o estado da arte no tocante ao emprego dos Sistemas de Recomendação no âmbito Educacional. Com isso, 
VIII Congresso Brasileiro de Informática na Educação (CBIE 2019)

Anais dos Workshops do VIII Congresso Brasileiro de Informática na Educação (WCBIE 2019)

procura identificar, a partir de alguns aspectos, como esse tema tem sido estudado pela comunidade acadêmica, além de indicar possíveis lacunas ainda pouco ou não exploradas.

Este trabalho se organiza como segue. A seção 2 apresenta a metodologia adotada para a realização desta pesquisa. A seção 3 apresenta os trabalhos resultantes da revisão, com um breve resumo de cada um deles. A seção 4 analisa e discute os trabalhos selecionados de acordo com alguns critérios definidos. Por fim, a seção 5 aponta alguns desafios presentes na área de estudo, bem como algumas possibilidades de pesquisa em aberto.

\section{Metodologia de Pesquisa}

Para o levantamento do estado da arte sobre o tema abordado, pesquisou-se por artigos que trabalhavam a temática de Sistemas de Recomendação no ambiente Educacional. As bases de busca primárias foram o IEEE Explore e Springer, além do Google Scholar, que foi utilizado subsidiariamente na identificação de outros trabalhos disponíveis não contemplados nessas bases e que pudessem ser relevantes para o objetivo desse trabalho.

Para cumprimento do objetivo de pesquisa supramencionado, os termos de busca utilizados foram "recommender system" e "educational", "recommender system" e "education", "recommender system" e "learning objects" e "learning objects recommender system". O período de busca foi estabelecido entre o ano de 2016 e o primeiro semestre de 2019. Com essa restrição de período, buscou-se concentrar no estágio bem atual da pesquisa nesse campo.

Estabelecidos os critérios e objetivos, passou-se ao processo de busca propriamente dito. A seleção dos artigos deu-se pela relação dos trabalhos com a temática pretendida pela presente revisão. Analisaram-se, num primeiro estágio, o título dos artigos, descartando aqueles se podia depreender não haver relação com o objeto da pesquisa. O critério de exclusão foi os trabalhos que não abordavam conjuntamente sistemas de recomendação e ambiente educacional. Essa etapa resultou em 21 artigos selecionados.

A seguir, passou-se à análise dos resumos e palavras-chave. Nessa fase, foram retirados 3 artigos cujo enfoque era revisão da literatura. A leitura do corpo do artigo foi a última etapa para eliminação dos trabalhos, realizada principalmente a partir dos objetivos, metodologia e ferramenta proposta, para selecionar aqueles que melhor contribuíam para o objetivo do presente trabalho. Esta etapa culminou na remoção de mais 5 artigos, que ofereciam poucas possibilidades de se identificar o mecanismo de recomendação ou o modo como ele foi aplicado.

Os 13 artigos resultantes foram analisados e agrupados segundo alguns critérios pré-definidos, a saber: abordagem e técnica de recomendação, estágio de aplicação do sistema (somente modelado, apenas implementado ou já aplicado) e disponibilidade da base de dados utilizada no experimento. Também foi analisado quais artigos utilizaram fundamentações teóricas da psicologia ou pedagogia. Por fim, a partir desses e de outros critérios, foram externados alguns desafios a serem enfrentados nessa área, bem como possibilidades em aberto a serem exploradas.

\section{Trabalhos Estudados}

Os Sistemas de Recomendação para fins Educacionais têm despertado interesse da comunidade científica. Todavia, ainda há caminhos a serem explorados. No processo de 
VIII Congresso Brasileiro de Informática na Educação (CBIE 2019)

Anais dos Workshops do VIII Congresso Brasileiro de Informática na Educação (WCBIE 2019)

revisão da literatura concentrada no período de 2016 a 2019, foram selecionados os artigos constantes na tabela 1 , os quais se encontram detalhados a seguir.

Tabela 1. Lista de trabalhos considerados na análise da literatura

\begin{tabular}{|l|c|}
\hline ID & Trabalhos \\
\hline$[1]$ & [Zhang et al. 2017] \\
\hline$[2]$ & [Imran et al. 2016] \\
\hline$[3]$ & [Hassan and Hamada 2016] \\
\hline$[4]$ & [Santos et al. 2016] \\
\hline$[5]$ & [Hassan and Hamada 2017] \\
\hline$[6]$ & [Gulzar et al. 2018] \\
\hline$[7]$ & [Dascalu et al. 2016] \\
\hline$[8]$ & [Sergis and Sampson 2016] \\
\hline$[9]$ & [Hoic-Bozic et al. 2016] \\
\hline$[10]$ & [Limongelli et al. 2016] \\
\hline$[11]$ & [Sweeney et al. 2016] \\
\hline$[12]$ & [Klašnja-Milićević et al. 2018] \\
\hline$[13]$ & [da S. Dias and Wives 2019] \\
\hline \hline
\end{tabular}

[Imran et al. 2016] propõem um Sistema de Recomendação de Objetos de Aprendizagem personalizado, que está integrado dentro de um Sistema de Gerenciamento de Aprendizagem (LMS) - Learning Management System. O sistema busca recomendar OAs a partir dos OAs que o próprio usuário visitou ou que outros estudantes com perfis semelhantes visitaram. Para se obter as informações de histórico de utilização e comportamento do usuário, são verificados os $\log s$ de acesso e utilização do sistema que contém a plataforma do curso. O sistema foi utilizado dentro de um curso, mas a eficácia das recomendações não foi mencionada.

[Hassan and Hamada 2016] exploram o fato de que os Sistemas de Recomendação Educacionais geralmente utilizam um único valor de avaliação do usuário para prever o seu grau de interesse no OA. Todavia, as preferências do usuário podem depender de mais de um tipo de atributo daquele OA, por exemplo, autor, preço e nível de dificuldade. Nesse contexto, os autores criam um Sistema de Recomendação multi-critério, que modela e estima as preferências do usuário usando não mais um único atributo de avaliação, mas sim um vetor, que contempla o valor de avaliação para cada atributo do OA. A técnica utiliza uma Rede Neural Artificial para a tarefa de aprender a recomendar. Os autores mencionam como próxima etapa do trabalho a integração do recomendador a um LMS.

[Santos et al. 2016] investigam como um Sistema de Recomendação auxilia os usuários a encontrarem OAs adequados. O repositório analisado é de uma instituição educacional brasileira, que usa o padrão de metadados OBAA. É proposto um algoritmo de recomendação baseado em conteúdo. Para tratar o problema e realizar cálculos de similaridade do conteúdos, são utilizados o Modelo de Espaço Vetor e o Raciocínio Baseado em Casos [Aamodt and Plaza 1994]. Os participantes da pesquisa relataram, em sua maioria, que as recomendações foram relativamente úteis ou significativamente úteis. Concluiu-se também que os usuários obtêm informações de modo mais rápido se souberem a qual assunto o OA está relacionado. 
VIII Congresso Brasileiro de Informática na Educação (CBIE 2019)

Anais dos Workshops do VIII Congresso Brasileiro de Informática na Educação (WCBIE 2019)

[Dascalu et al. 2016] apresentam um Sistema de Recomendação baseado no poder semântico das ontologias. Inicialmente, a ontologia é povoada com instâncias (dados) de comunidades, interesses, comunidade de aprendizes, recrutadores, cargos e habilidades, obtidos a partir da plataforma Linkedin. Os dados relevantes são extraídos e armazenados em um grafo de ontologias. Os cargos ocupados e habilidades do usuário são explorados para fazer inferências e encontrar comunidades que possuem os mesmos interesses, apoiando tanto o aprendizado durante o curso de graduação como a inserção no meio profissional. Foram realizados testes preliminares do sistema, onde foram obtidos resultados satisfatórios, além de identificar limitações que precisam ser corrigidas no futuro.

[Sergis and Sampson 2016] almejam auxiliar os professores na seleção de OAs de repositórios existentes. Para tal, o sistema faz a recomendação em duas etapas: primeiro, por meio da construção automática dos Perfis de Competências Digitais dos professores, a qual se baseia nas ações dos mesmos dentro dos sistemas dos diferentes repositórios. Em seguida, esses perfis criados são explorados para se fazer uma seleção de OAs mais eficiente. Foram apresentados experimentos com 3 repositórios reais. Os resultados das avaliações demonstram que a abordagem proposta fornece recomendações com maior acurácia aos professores, baseadas nos seus perfis.

[Hoic-Bozic et al. 2016] propõem um modelo de aprendizagem (blended learning) que combina um LMS, um conjunto de ferramentas Web 2.0 e um Sistema de Recomendação de Atividades de e-learning (ELARS). Para tal, o modelo de aprendizado combinado foi implementado e avaliado dentro do curso "Hypermidia Supported Education”. Os estudantes que utilizaram o recomendador alcançaram resultados melhores em relação aos que não o utilizaram, não apenas por resultados finais, mas também por atividade. Em questionários anônimos, os alunos expressaram satisfação sobre a efetividade do modelo de aprendizado combinado e apontaram o ELARS um sistema útil.

[Limongelli et al. 2016] desenvolvem um Sistema de Recomendação voltado para os professores, no intuito de ajudá-los a montar cursos a partir de pesquisas por OAs nos repositórios mais populares. A montagem dos cursos é possibilitada a partir do sistema Moodle. O mecanismo de recomendação dá prioridade para OAs que foram escolhidos por outros usuários, em uma abordagem colaborativa. Aplicado em uma amostra de 25 professores, o sistema reduziu o tempo necessário para obter os objetos de aprendizagem nos repositórios, sendo apontado como útil por $80 \%$ dos participantes do experimento.

[Sweeney et al. 2016] desenvolvem um Sistema de Recomendação que auxilia o aluno, a partir do seu desempenho, na escolha semestral das disciplinas a serem cursadas no curso de graduação. Os autores buscam técnicas de recomendação empregadas no e-commerce para serem utilizadas no monitoramento do progresso do aluno e processo de recomendação, procurando identificar aspectos que contribuam para a aprovação do estudante. O melhor resultado dentre vários algoritmos estudados foi obtido por uma abordagem híbrida entre o Factorization Machines (FM) e Random Forests (RF). O conjunto de estudantes utilizados no experimento foi da Universidade de George Mason.

[Zhang et al. 2017] propõem um algoritmo de recomendação para ambientes de MOOCs (massive online open courses). Para lidar com a esparsidade e grande volume de dados que esses ambientes de MOOCs possuem, é proposta uma abordagem baseada em redes neurais profundas. $\mathrm{O}$ modelo é utilizado para extrair as características do conjunto 
VIII Congresso Brasileiro de Informática na Educação (CBIE 2019)

Anais dos Workshops do VIII Congresso Brasileiro de Informática na Educação (WCBIE 2019)

de dados da plataforma Starc, tendo a nota do curso dos alunos como o rótulo da classe de aprendizado supervisionado. A proposta obteve melhores resultados que outros métodos, tais como recomendação baseada em conteúdo e o método da descida gradiente.

[Hassan and Hamada 2017] exploram o fato de que a maioria dos sistemas não considera informações de contexto na qual a recomendação se dá. Para lidar com esse problema, é proposta uma arquitetura de sistema que lida com informações contextuais, a ser utilizada por meio de dispositivos inteligentes como smartphones, para fazer as recomendações. É proposto um framework conceitual que pode ser executado tanto em iOS quanto em dispositivos Android. O sistema proposto, ainda apenas um modelo, utiliza o conceito de Estilos de Aprendizagem como informação contextual para melhorar as recomendações de OAs.

[Gulzar et al. 2018] apresentam um Sistema de Recomendação de cursos, construído numa abordagem híbrida que utiliza técnicas de recuperação da informação, acrescidas do auxílio de ontologia. Dentro de um conjunto de cursos são realizadas consultas que passam a ser convertidas em n-gramas, sobre os quais é utilizada a ferramenta WordNet para recuperação de palavras similares. Em outra etapa são removidas possíveis redundâncias até que por fim são extraídos cursos relacionados em um repositório com o auxílio da ontologia de curso. A acurácia das recomendações foi testada em disciplinas de um curso em uma Universidade, saindo-se melhor em comparação com outras técnicas.

[Klašnja-Milićević et al. 2018] utiliza um recomendador baseado em tags para Ambientes Virtuais de Aprendizagem, em que tags são atribuídas aos Objetos de Aprendizagem. A abordagem foi avaliada no sistema de tutoria Protus, onde participaram 120 alunos do ensino médio. Foi utilizada uma técnica de clusterização para agrupar os participantes a partir de Estilos de Aprendizagem para reduzir a necessidade de processamento e memória. Ao se analisar vários algoritmos para recomendação no experimento, a técnica que obteve melhores resultados foi através de fatoração tensorial.

[da S. Dias and Wives 2019] utilizam uma proposta a ser aplicada no contexto de sistemas de e-learning ubíquos, que possibilitam os estudantes interagirem de várias formas no processo de aprendizagem. Para o mecanismo de recomendação, é utilizada a busca do vizinho mais próximo baseada no usuário, que é alimentada com informações a partir do comportamento do usuário em suas múltiplas interações no sistema. O desenvolvimento do sistema se fundamenta também no princípio do aluno como aquele que controla seu próprio aprendizado. Testes realizados com alunos da UFRGS na plataforma AdaptWeb mostraram a abordagem proposta com melhores resultados na recomendação.

\section{Análise dos Trabalhos}

As tabelas 2, 3 e 4 evidenciam alguns aspectos analisados nos trabalhos.

A tabela 2 divide os trabalhos de acordo com a técnica que foi empregada no processo de recomendação. Boa parte dos Sistemas de Recomendação utiliza abordagens híbridas, isto é, estratégias combinadas, com a intenção de explorar as potencialidades e atenuar as fraquezas de cada uma das técnicas, como por exemplo, Ontologia e Filtragem Colaborativa, utilizadas em um mesmo sistema.

A tabela 3 apresenta quais trabalhos apenas propuseram o sistema, em comparação com aqueles que o aplicaram em alguma base de dados e, ou conjunto de pessoas. Mais 
VIII Congresso Brasileiro de Informática na Educação (CBIE 2019)

Anais dos Workshops do VIII Congresso Brasileiro de Informática na Educação (WCBIE 2019)

Tabela 2. Abordagem/técnica empregada no Sistema de Recomendação.

\begin{tabular}{|l|c|}
\hline Técnica & Trabalhos \\
\hline Filtragem Colaborativa & {$[10]$} \\
\hline Baseada em Conteúdo & {$[4]$} \\
\hline Baseada em Ontologia & {$[7]$} \\
\hline Lógica Fuzzy & {$[8]$} \\
\hline Redes neurais & {$[1],[3]$} \\
\hline Híbrida & {$[2],[5],[6],[9],[11]$} \\
\hline Outras & {$[12],[13]$} \\
\hline \hline
\end{tabular}

da metade dos trabalhos publicados de fato aplicam o sistema proposto. Há uma menor porção de trabalhos que ainda não testou o desempenho do seu recomendador em uma situação real, que possa ser comparável a outras técnicas já empregadas.

Tabela 3. Estágio de Aplicação dos Sistemas de Recomendação.

\begin{tabular}{|l|c|}
\hline Estágio & Trabalhos \\
\hline Apenas modelagem (conceitual) & {$[3],[5],[7]$} \\
\hline Implementado, mas não aplicado & {$[2]$} \\
\hline Aplicado & {$[1],[4],[6],[8],[9],[10],[11],[12],[13]$} \\
\hline
\end{tabular}

A tabela 4 separa os trabalhos de acordo com as bases de dados utilizadas para testar o Sistema de Recomendação: se a base de dados está disponível publicamente, se é restrita ao pesquisador que fez o estudo, ou se não é utilizada - caso dos trabalhos que apenas propõem um sistema, mas não o colocaram em prática até então. Percebe-se que a maioria das bases de dados são restritas e pelo que pode se depreender são pequenas, o que dificulta a comparação de um sistema proposto com outros já utilizados, ou mesmo com a recomendação avaliada pelos usuários no mundo real.

Tabela 4. Base de dados utilizada para teste.

\begin{tabular}{|l|c|}
\hline Tipo & Trabalhos \\
\hline Público & {$[1],[8]$} \\
\hline Restrito & {$[4],[6],[9],[10],[11],[12],[13]$} \\
\hline Não utiliza & {$[2],[3],[5],[7]$} \\
\hline \hline
\end{tabular}

Por fim, a tabela 5 distingue aqueles trabalhos cujo Sistema de Recomendação foi elaborado considerando algum conceito teórico da psicologia ou pedagogia. Conforme mostra a tabela, a maioria dos trabalhos não tem como elemento balizador de seu sistema as fundamentações teóricas advindas seja da psicologia ou pedagogia, as quais são elementos norteadores e importantes no processo de ensino-aprendizagem.

Dentre os trabalhos que se valeram dessas teorias, [Imran et al. 2016] utilizam o modelo de Felder-Silverman [Felder et al. 1988] para detecção do Estilo de Aprendizagem dos alunos e sua utilização no processo de recomendação. [Hassan and Hamada 2017] também utilizam esse modelo, porém com a finalidade de 
VIII Congresso Brasileiro de Informática na Educação (CBIE 2019)

Anais dos Workshops do VIII Congresso Brasileiro de Informática na Educação (WCBIE 2019)

modelar informação contextual do Sistema. [Klašnja-Milićević et al. 2018] se vale do mesmo modelo para agrupar os estudantes e reduzir a necessidade de processamento. [Hoic-Bozic et al. 2016] se vale dos princípios do Processo de Bologna [Keeling 2006], uma iniciativa da Comissão Europeia para promover uma reforma educacional.

Tabela 5. Utilização de fundamentação teórica psicopedagógica.

\begin{tabular}{|l|c|}
\hline Utilização de teoria & Trabalhos \\
\hline Utiliza & {$[2],[5],[9],[12],[13]$} \\
\hline Não utiliza & {$[1],[3],[4],[6],[7],[8],[10],[11]$} \\
\hline
\end{tabular}

\section{Conclusão e Perspectivas futuras}

A partir da análise realizada, não restrita apenas aos aspectos ora enfatizados, pode-se notar alguns aspectos relacionados ao conjunto dos trabalhos, os quais são listados a seguir.

- Não existem muitos trabalhos nos quais as recomendações levam em consideração ambas as preferências de alunos e tutores/professores;

- A maior parte dos trabalhos não possui alguma fundamentação teórica advinda seja da psicologia ou mesmo da pedagogia;

- A maioria dos trabalhos publicados aplicam o sistema proposto. No entanto, as bases de dados utilizadas geralmente são restritas e ao que tudo indica são pequenas, o que dificulta a comparação de um sistema proposto com outros já utilizados, ou mesmo com a recomendação avaliada pelo usuário no mundo real;

- A maioria dos Sistemas de Recomendação utiliza abordagens híbridas, isto é, estratégias combinadas, com a intenção de explorar as potencialidades e atenuar as fraquezas de cada uma das técnicas.

Tais constatações indicam algumas possibilidades e desafios a serem explorados nessa área. A primeira seria a necessidade da existência de conjuntos de dados robustos e públicos, específicos da área educacional, que possibilitassem a comparação entre diferentes técnicas quanto a sua acurácia nas recomendações. Há bases de dados disponíveis dessa natureza como a Movielens [Grouplens 2018] e Last.fm [Kaggle 2019], porém as mesmas não são específicas ao contexto educacional.

Outro ponto importante é que o processo de ensino-aprendizagem é complexo, envolvendo diversos estudos teóricos e práticos de várias áreas do conhecimento, como a psicologia e a pedagogia. É importante haver mais sistemas de recomendação que levem em consideração esses aspectos, de modo a torná-los uma ferramenta mais integrada ao processo educacional como um todo. Sistemas que trabalhem com propostas pedagógicas e curriculares específicas, como metologias ativas [Berbel 2011] e pensamento computacional [Wing 2006], são possibilidades aventadas.

Com relação à abordagem para implementação dos sistemas, uma seara a ser melhor explorada é a de Sistemas de Recomendação multiobjetivo. Embora existam trabalhos na área, como [Zuo et al. 2015], [Wang et al. 2016] e [Ribeiro et al. 2014], eles não tratam de modo específico o contexto educacional, que tem as suas particularidades. Há muitas situações conflitantes nesse contexto que poderiam ser tratadas numa abordagem 
VIII Congresso Brasileiro de Informática na Educação (CBIE 2019)

Anais dos Workshops do VIII Congresso Brasileiro de Informática na Educação (WCBIE 2019)

multiobjetivo, por exemplo, as preferências dos alunos e dos seus professores, a escolha entre o OA mais popular e outro pouco conhecido, mas que teria potencial utilização.

As análises realizadas mostram a importância dos Sistemas de Recomendação no novo paradigma educacional do e-learning, que tem crescido vertiginosamente. Foi possível identificar desafios a serem superados na área para que se realize uma melhor comparação entre os diferentes trabalhos conduzidos pela comunidade científica. Também foram identificadas possibilidades a serem exploradas nesse domínio da Educação, que demonstra ser esse um campo promissor e em crescimento.

\section{Referências}

Aamodt, A. and Plaza, E. (1994). Case-based reasoning: Foundational issues, methodological variations, and system approaches. AI communications, 7(1):39-59.

Berbel, N. A. N. (2011). As metodologias ativas e a promoção da autonomia de estudantes. Semina: Ciências Sociais e Humanas, 32(1):25-40.

Bobadilla, J., Ortega, F., Hernando, A., and Gutiérrez, A. (2013). Recommender systems survey. Knowledge-Based Systems, 46:109-132.

D C M, I. and Others (2008). Dcmi metadata terms. World Wide Web electronic publication.

da S. Dias, A. and Wives, L. K. (2019). Recommender system for learning objects based in the fusion of social signals, interests, and preferences of learner users in ubiquitous e-learning systems. Personal and Ubiquitous Computing, 23(2):249-268.

Dascalu, M.-I., Bodea, C.-N., Mihailescu, M. N., Tanase, E. A., and Ordoñez de Pablos, P. (2016). Educational recommender systems and their application in lifelong learning. Behaviour \& Information Technology, 35(4):290-297.

Felder, R. M., Silverman, L. K., et al. (1988). Learning and teaching styles in engineering education. Engineering education, 78(7):674-681.

Grouplens (2018). Movielens Latest Datasets.

Gulzar, Z., Leema, A. A., and Deepak, G. (2018). PCRS: Personalized Course Recommender System Based on Hybrid Approach. Procedia Computer Science, 125:518524.

Hassan, M. and Hamada, M. (2016). Enhancing learning objects recommendation using multi-criteria recommender systems. In 2016 IEEE International Conference on Teaching, Assessment, and Learning for Engineering (TALE), pages 62-64. IEEE.

Hassan, M. and Hamada, M. (2017). Smart media-based context-aware recommender systems for learning: A conceptual framework. In 2017 16th International Conference on Information Technology Based Higher Education and Training (ITHET), pages 1-4. IEEE.

Hoic-Bozic, N., Holenko Dlab, M., and Mornar, V. (2016). Recommender System and Web 2.0 Tools to Enhance a Blended Learning Model. IEEE Transactions on Education, 59(1):39-44.

IEEE (2002). Learning Technology Standards Committee (LTSC). 
VIII Congresso Brasileiro de Informática na Educação (CBIE 2019)

Anais dos Workshops do VIII Congresso Brasileiro de Informática na Educação (WCBIE 2019)

Imran, H., Belghis-Zadeh, M., Chang, T.-W., Kinshuk, and Graf, S. (2016). PLORS: a personalized learning object recommender system. Vietnam Journal of Computer Science, 3(1):3-13.

IMS (2019). Ims global learning consortium.

Kaggle (2019). last.fm dataset - Kaggle.

Katarya, R. and Verma, O. P. (2018). Recommender system with grey wolf optimizer and FCM. Neural Computing and Applications, 30(5):1679-1687.

Keeling, R. (2006). The bologna process and the lisbon research agenda: the european commission's expanding role in higher education discourse. European journal of education, 41(2):203-223.

Klašnja-Milićević, A., Ivanović, M., Vesin, B., and Budimac, Z. (2018). Enhancing elearning systems with personalized recommendation based on collaborative tagging techniques. Applied Intelligence, 48(6):1519-1535.

Limongelli, C., Lombardi, M., Marani, A., Sciarrone, F., and Temperini, M. (2016). A recommendation module to help teachers build courses through the Moodle Learning Management System. New Review of Hypermedia and Multimedia, 22(1-2):58-82.

Ribeiro, M. T., Ziviani, N., Moura, E. S. D., Hata, I., Lacerda, A., and Veloso, A. (2014). Multiobjective Pareto-Efficient Approaches for Recommender Systems. ACM Transactions on Intelligent Systems and Technology, 5(4):1-20.

Ricci, F., Rokach, L., and Shapira, B. (2015). Recommender Systems: Introduction and Challenges. In Recommender Systems Handbook, pages 1-34. Springer US, Boston, MA.

Santos, M., Andrade, F., da Silva, J. M. C., and Imran, H. (2016). Learning Object Recommendation System Evaluation. In 2016 IEEE 16th International Conference on Advanced Learning Technologies (ICALT), pages 412-413. IEEE.

Sergis, S. and Sampson, D. G. (2016). Learning Object Recommendations for Teachers Based On Elicited ICT Competence Profiles. IEEE Transactions on Learning Technologies, 9(1):67-80.

Sweeney, M., Rangwala, H., Lester, J., and Johri, A. (2016). Next-Term Student Performance Prediction: A Recommender Systems Approach.

Wang, S., Gong, M., Li, H., and Yang, J. (2016). Multi-objective optimization for long tail recommendation. Knowledge-Based Systems, 104:145-155.

Wing, J. M. (2006). Computational thinking. Communications of the ACM, 49(3):33-35.

Yao, X. (1999). Evolving artificial neural networks. Proceedings of the IEEE, 87(9):1423-1447.

Zhang, H., Yang, H., Huang, T., and Zhan, G. (2017). DBNCF: Personalized Courses Recommendation System Based on DBN in MOOC Environment. In 2017 International Symposium on Educational Technology (ISET), pages 106-108. IEEE.

Zuo, Y., Gong, M., Zeng, J., Ma, L., and Jiao, L. (2015). Personalized Recommendation Based on Evolutionary Multi-Objective Optimization [Research Frontier]. IEEE Computational Intelligence Magazine, 10(1):52-62. 